Feasibility of Sea-level

Cosmic-Ray Muon-Capture SNM Detection

L. Rosenberg, A. Bernstein

March 14, 2005 
This document was prepared as an account of work sponsored by an agency of the United States Government. Neither the United States Government nor the University of California nor any of their employees, makes any warranty, express or implied, or assumes any legal liability or responsibility for the accuracy, completeness, or usefulness of any information, apparatus, product, or process disclosed, or represents that its use would not infringe privately owned rights. Reference herein to any specific commercial product, process, or service by trade name, trademark, manufacturer, or otherwise, does not necessarily constitute or imply its endorsement, recommendation, or favoring by the United States Government or the University of California. The views and opinions of authors expressed herein do not necessarily state or reflect those of the United States Government or the University of California, and shall not be used for advertising or product endorsement purposes.

This work was performed under the auspices of the U.S. Department of Energy by University of California, Lawrence Livermore National Laboratory under Contract W-7405-Eng-48. 


\title{
FEASIBILITY OF SEA-LEVEL COSMIC-RAY MUON-CAPTURE SNM DETECTION
}

\author{
Leslie Rosenberg and Adam Bernstein \\ Lawrence Livermore National Laboratory, \\ Physics and Advanced Technologies Directorate
}

January 2005

INTRODUCTION

Interest has lately risen in the idea of detecting the energetic muonic X-rays from cosmic-ray capture in highly enriched uranium (HEU) or plutonium (Pu). The basic problem is that HEU or $\mathrm{Pu}$ (collectively special nuclear material: SNM) could be smuggled into our country within a cargo container. Since at present, only a small fraction of the millions of entering cargo containers are subjected to any serious scrutiny, the odds are in favor of the SNM arriving undetected. A major program is underway, which will likely expand greatly, to develop portal monitoring for SNM in cargos. The muon-capture idea, recently reintroduced by Chuck Hailey (Columbia), ${ }^{1}$ is to detect $\mathrm{MeV}$ gamma rays emerging from the interaction of the SNM with cosmic ray negatively-charged muons as the signature of SNM.

In more detail, the interaction is for the negative muons (simply "muons" in what follows) to be captured in SNM atomic orbitals. As the muon cascades down atomic levels, it emits characteristic x-rays. Owing to the large mass of the muon and the large nuclear charge of HEU and $\mathrm{Pu}$, the $\mathrm{x}$-rays for low-lying transitions have MeV energies (we loosely call these gamma rays).

This strikes some people as a promising technique since the cosmic ray muons are very penetrating as are the multi-MeV muonic-atom X-rays; the muon

\footnotetext{
${ }^{1}$ There could well be antecedents to Hailey's idea
} 
probe penetrates the cargo, and the muonic gamma rays can fairly easily escape. Early estimates gave low signal rates for detecting these gamma rays; say, the proponents hoped, on order of several per minute. The low rate was discouraging. However, the proponents advanced the argument that since background multi-MeV gamma rays are somewhat rare, and the signal gamma rays are distinctive in energy and in their coincidence with a cosmic-ray muon, even a few number of events, perhaps even just one, offers the possibility of a high confidence SNM detection. That muons and gammarays are so penetrating implies this signature would be considerably harder to defeat than that from other passive approaches. A further benefit of this approach is removing the need for an active particle probe beam with its necessary accelerator components and radiation dose. Hence, the great interest by some in the muon-capture idea.

In an earlier study, we reached a preliminary conclusion ${ }^{2}$ that the signal rate will be lower than hoped for, and the signal gamma rays could be diluted by muogenic backgrounds when realistic conditions are applied. This note elaborates on those earlier conclusions. In particular for the signal, we estimate the signal rate for that part of the cosmic ray negative muon energy spectrum participating in capture and gamma-ray escape, finding the average time between usable captures is several minutes to several tens of minutes. We also estimate the energy spectrum of the escape signal gamma rays, finding it substantially broadened by the splittings of the low-lying muogenic SNM atomic levels. We also estimate one class of background, that of the photon halo surrounding the muon inducing nuclear breakup in the detector media itself. There are other backgrounds (e.g., muogenic isotope activation) but this first background alone is significant over the

\footnotetext{
${ }^{2}$ Internal LLNL note to Bill Goldstein, September 200.
} 
large energy width of the signal gamma rays. This background is intrinsic to the technique, and therefore is a largely irreducible background in any practical detector.

\section{MUON CAPTURE RATES}

Gamma-rays do not have high penetrating power in SNM. This can be seen from Figure 1 and Table 1, the mass-density normalized gamma-ray exponential attenuation coefficient as a function of gamma-ray energy. The attenuation length rapidly increases (inverse coefficient falls) as the gamma-ray energy increases into the $\mathrm{MeV}$ range. Above $1 \mathrm{MeV}$ gammaray energy, the gamma-ray attenuation is more or less flat. For example: for a capture X-ray of energy $3 \mathrm{MeV}$ (an energy below which terrestrial backgrounds grow sharply) to have a reasonable chance of escape unscathed, the gamma ray can't traverse more than about $1 \mathrm{~cm}$ before leaving the SNM, and even then, it needs to propagate towards the surface, not tangentially or inwards. We carry forward this characteristic attenuation length of 1 $\mathrm{cm}$ for the muonic-atom x-rays.

The penetrating power of muons is high, so most muons will penetrate much deeper than $1 \mathrm{~cm}$ into the material. ${ }^{3}$ Since the total cosmic-ray muon rate at sea level through the palm of your hand is about a Hertz, you can see already that the capture rate will be small for realistic target volumes. The energy and material dependence of the muon range is shown in Figure 3 in units of target mass density times projectile mass. The highest- $z$ material shown is lead, but the high-z materials bunch near the same asymptote on this figure and is a good approximation for the range in SNM. The range decreases as the muon energy decreases. When the muon momentum approaches the muon mass, the range

\footnotetext{
${ }^{3}$ It's possible a higher-energy muon will penetrate through the SNM and stop within 1 $\mathrm{cm}$ of the far surface. We comment on this later.
} 
decreases sharply due the greatly increased muon stopping power. ${ }^{4}$ The range of muons of energy 200 $\mathrm{MeV} / \mathrm{C}$ is $4.5 \mathrm{~cm}$. The range of muons of momentum $100 \mathrm{MeV} / \mathrm{c}$ is $1.1 \mathrm{~cm}$ : the energy width of usable cosmic-ray muon energies is therefore around 100 $\mathrm{MeV}$, while most of the other cosmic rays do not result in a detectable gamma-ray signature. The sea-level cosmic-ray muon energy spectrum is shown in Figure 3, scaled by a power of the muon momentum to flatten the curve; the flux is relatively flat for muon momenta below about $2 \mathrm{GeV} / \mathrm{c}$. The solid points are for vertical muons, the hollow points are for steeply-arriving muons (through more atmosphere). Notice adding overburden hardens the spectra, reducing the overall number of muons overall and significantly depleting the lowmomentum component. There are published measurements of lower-momenta sea level muon spectra, but such measurements are rare and typically not carefully done. However, for muon momenta of $200 \mathrm{MeV}$ and greater, there is a recent publication "CAPRICE94" of the sea-level muon flux, separated into charge components; this is tabulated in Table 2. We take the lowest momentum bin for our characteristic negative muon flux; the distribution is fairly flat in this region. At the lowest momentum bin (200-300 MeV/c), the negative muon flux in a $100 \mathrm{MeV}$ energy interval over $\pi \mathrm{sr}$ is less than $10 \mathrm{~Hz} / \mathrm{m}^{2}$ sec. Even with a SNM target cross section of $100 \mathrm{~cm}^{2}$, the inverse rate of usable low energy negatively-charged cosmic ray muons entering the target is on order of a minute. We can also at this point make a crude estimate of the maximum muon-capture rate resulting from a higher momentum muon traversing the target and losing just enough energy to stop within $1 \mathrm{~cm}$ of the opposite surface. We arrive at this by taking the maximum flux from Table 2 and otherwise applying the same conditions. This is easy to do since the flux is more or less flat and near

\footnotetext{
${ }^{4}$ Unfortunately the range of pure negative muons is less due to nuclear-capture.
} 
maximum from $200 \mathrm{MeV} / \mathrm{c}$ to a few $\mathrm{GeV} / \mathrm{c}$; the result is the same as from the previous calculation. Therefore, there is no great gain to be had in considering the stopping rate for these highermomentum muons. Nor can unusual SNM target shapes sharply increase the muon-stopping rate. We also note that not all the stopping-muon gamma rays will escape the target, only those leaving the near surface will escape. This geometric correction is highly shape dependent. For a spherical $20 \mathrm{~kg}$ HEU ball, each multi-MeV gamma ray from the optimal 100 $\mathrm{MeV}$ muon momentum band has about a one in three chance of escape. For a $20 \mathrm{~kg}$ flat $1 \mathrm{~cm}$ thick plate of HEU, each multi-MeV gamma ray has about a two in three chance of escape. The conclusion is that only a small fraction of the incident cosmic ray flux will stop near enough to the surface.

\section{MUONIC X-RAY ENERGY SPECTRA AND LINE INTENSITY}

A naive extrapolation of hydrogen Rydberg electronic energy levels to muonic atom levels in SNM predicts X-rays of MeV energies, with transitions to the $1 \mathrm{~s}, 2 \mathrm{~s}$ and $3 \mathrm{~s}$ muonic states in HEU having energy differences 18,3 and $1 \mathrm{MeV}$. Although the $1 \mathrm{MeV}$ gamma rays would be very hard to detect in a background sea of such gamma rays, the two higher energy gamma rays are somewhat rare in the background gamma ray sea, are correlated in time with the stopped muon, and are distinctive in energy. The proponents assert this signature is backgroundless.

The muonic atom actinide $\mathrm{X}$-ray spectra are well known from measurements with high-resolution gammaray counters and muon beams; the original motivation in studying the gamma-ray energy spectrum was to probe nuclear structure. As you might expect, the measured low-lying muonic levels are considerably more complicated than that of a simple extrapolation from hydrogen. The measured 
muonic $\mathrm{X}$-ray energies and relative intensities from HEU for the lowest two sets of transitions (the highest energy transitions) are shown in Table 3, along with two theoretical estimates. Notice the energies of these inner level transitions are highly perturbed. The 2p-1s transition is around 6 $\mathrm{MeV}$, the $3 \mathrm{~d}-2 \mathrm{p}$ transition is around $3 \mathrm{MeV}$. The table doesn't show the $4 \mathrm{f}-3 \mathrm{~d}$ transitions, but they are around $1 \mathrm{MeV}$, close to their unperturbed energy. There are no $15 \mathrm{MeV}$ gamma rays. Also note there is considerable fine and hyperfine splitting of the levels. For instance, the 2p-1s transition is distributed among states over a $500 \mathrm{keV}$ energy interval. Hence, high-resolution detectors don't significantly increase the signal-to-noise ratio. Figure 4 shows the measured $2 \mathrm{p}-1 \mathrm{~s}$ and $3 \mathrm{~d}-2 \mathrm{p}$ muonic $\mathrm{X}$-ray spectra (from Table 3 ) for a range of actinides, including $\mathrm{HEU}$ and $\mathrm{Pu}$. The complicated structure and wide energy range of the X-rays is apparent. The yields for the quadrupole and higher-order transitions, some with higher energy $\mathrm{x}$-rays, are very small.

At this point, we have the key result that the signal gamma-ray rate is low, and the $\mathrm{X}$-ray lines are severely split. Therefore, a detector of these $\mathrm{X}$-rays needs a large solid angle acceptance over a large area (the surface of a cargo container). Since having high-resolution isn't a great advantage, the obvious detector technology is large volumes of hydrocarbon scintillator (plastic or liquid). A large path length (depth), and therefore a large detector volume is needed to have a high efficiency for having the muonic $x$-rays interact in the detector. The probability of both higher-energy X-rays escaping from the spherical SNM target of the last section was about $1 / 10$, so the time required for a high-confidence two-X-ray detection (assuming no background) is tens of minutes. Probably this is too long for the program goals. If instead, only the highest energy $\mathrm{X}$-ray 
is detected, the time required for a highconfidence one-X-ray detection (assuming no background) is a few minutes; longer than the program goals, but within a factor of a few. At 6 $\mathrm{MeV}$, there aren't a huge number of environmental gamma rays; there is considerably more background at $3 \mathrm{MeV}$. If these environmental backgrounds dilute the signal, very much more time will be required for a significant detection. The conclusion is that the detected coincidence of the two most energetic $\mathrm{X}$-rays is rare so the time for detection is much longer than the program goals. However, if only the most energetic x-ray is detected, the rate approaches within a factor of a few of the program goals, assuming no background counts.

\section{MUOGENIC BACKGROUNDS}

The rate for cosmic rays traversing a sea-level cargo container is on order of $10 \mathrm{kHz}$. Probably, a one microsecond time window is sufficient to associate a detected gamma ray with a cosmic muon. This timing window can in principle reduce the background gamma ray contamination to low levels, unless the backgrounds are themselves correlated with cosmic rays. There are two broad classes of such correlated muogenic backgrounds. The first class are products of muons activating materials in the cargo, detector and environment. These isotopes decay (some promptly, some very slowly) with the electromagnetic decay products are backgrounds to the muonic X-ray signal. Surprisingly little is known about the activation yields for cosmic rays with characteristic sealevel muon energies; the beta backgrounds vary considerably from place to place. Much more is known about activation of hydrocarbon materials for the harder muon spectra found deep underground, ${ }^{5}$ since deep-underground detectors have very low

\footnotetext{
${ }^{5}$ See, e.g., T. Hagner et al., Astroparticle Phys. 14 (2000) 33.
} 
background requirements. The second class of muogenic backgrounds are products of interactions of the virtual photon halo surrounding the cosmicray muon interacting with cargo, detector and environment. Of this background, the most pernicious is that associated with the virtual photon breaking up a nucleus and releasing neutrons. The neutron then strikes the hydrogen in the hydrocarbon scintillator itself, thereby causing a recoil proton to be detected as a background. The detector itself is the target, and the recoil proton (the background) is in-time with the muon and detected by the target itself.

Again, less is known about the neutron yield of cosmic-ray muons at shallow depths (soft muon energy spectrum) than at great depths (harder spectrum). However, what information there is on neutron yields at shallow sites is broadly consistent within a factor of three. ${ }^{6}$ Much interest in these neutron backgrounds comes from the microchip fabrication community, which worries about neutron-induced semiconductor faults. Figure 5 shows the total neutron yield at various depths (neutrons per cosmic ray per target path mass) from data of Boehm et al. The target in this work is liquid scintillator, so the results of this study are particularly germane. As expected, the yield grows with increasing average muon energy. The Boehm et al. result gives about 3 neutrons per muon per $\mathrm{g} / \mathrm{cm}^{2}$ of target mass. Extrapolating this to the surface from their $32 \mathrm{mWE}$ overburden gives a surface neutron yield of about one neutron per muon per $\mathrm{g} / \mathrm{cm}^{2}$ of scintillator target mass (within orders of magnitude). From modeling with FLUKA, almost all of these neutrons are products of photonuclear disintegration caused by the virtual photon cloud around the muon. Boehm et al. did not

\footnotetext{
${ }^{6}$ Y. -F. Wang, et al., Phys. Rev. D 64 (2001) 013012 (theory) and F. Boehm et al., Phys. Rev. D 62 (2000) 092005.
} 
measure the neutron energy spectrum, just the total neutron yield.

There is no agreement on the neutron energy spectrum. However, we are interested in characteristic values of energy spectra. For this, we can appeal to near sea-level measurements. The Karmen Detector ${ }^{7}$ results are particularly useful as they measure relatively low neutron energies and they provided a useful and simple parameterization of the neutron energy spectrum. They suggest an exponential energy distribution $\operatorname{Exp}\left(-\mathrm{E}_{\mathrm{n}} / 39 \mathrm{MeV}\right)$, where the normalization comes from the total neutron yield. Figure 6 shows this Karmen distribution normalized to the sea-level extrapolation of the Boehm et al. neutron yield. Notice this process generates copious amounts of fast neutrons.

These neutrons can then strike protons in the hydrocarbon scintillator thereby inducing proton recoils. The kinematics is that of billiard-balls: isotropic s-wave scattering in the center-of-mass system. The resulting proton recoil spectrum is shown in Figure 7. Notice the reasonably large yield of multi-MeV proton recoils.

As mentioned earlier, likely the detector media will be hydrocarbon scintillator of approximate density $1 \mathrm{~g} / \mathrm{cm}^{3}$. The energy resolution for such a detector is in the neighborhood of $20 \%$. Hence, for the higher energy muon capture gamma ray of energy $6 \mathrm{MeV}$, a high-efficiency detection would require a $1.5 \mathrm{MeV}$ energy window. Integrating the Figure 7 yield at $6 \mathrm{MeV}$ within this $1.5 \mathrm{MeV}$ window gives a yield 0.05 protons $/\left(\mathrm{g} / \mathrm{cm}^{2}\right.$ muon $)$. We assume the depth of the liquid scintillator detector is $20 \mathrm{~cm}$; it has to be fairly deep to ensure it has high efficiency to detect the full gamma-ray energy and this amount of scintillator is also has a

\footnotetext{
${ }^{7}$ See, e.g., the overview in the Boehm et al. paper.
} 
reasonably high efficiency for the neutron to scatter. The proton yield through the one side of the detector is therefore around 1 background protons per cosmic ray muon. Given that the muon crossing rate is measured in $\mathrm{kHz}$, this is potentially a large source of backgrounds.

\section{SUMMARY}

The first part of this report argues the average time between signal events for X-rays from negative muon capture on SNM is from a few to a few 10 's of minutes, depending on how sophisticated one care's to make the detector. The second part of this report argues that the recoil proton background in the energy resolution window can be orders of magnitude larger than the expected signal. How could one evade this result? Firstly, one could conceive of a very highly segmented muon counter (or electromagnetic calorimeter) system to actually detect a stopping muon. This would be extraordinarily expensive for a large area and volume of a cargo container. There are also quite a few assumptions we applied to make the calculations tractable. For instance, we assumed the detector was fully efficient for a neutron recoil. probably something like $25 \%$ or $50 \%$ is more appropriate. However, probably the biggest uncertainty is the neutron energy spectrum. The Boehm et al. paper discusses the range of spectrum parameterizations, some of which are considerably softer and will lower the high-energy proton yield. This outcome is certainly possible. However, given the difference between signal and background rates, it would take a considerable change in detector parameters and particle yields to change the basic conclusion that this technique does not appear promising. 


\begin{tabular}{ccc}
\hline $\begin{array}{c}\text { Energy } \\
(\mathrm{MeV})\end{array}$ & $\mu$ & $\begin{array}{c}\mu_{\mathrm{en}} \\
(\mathrm{cm} 2 / \mathrm{g})\end{array}$ \\
\hline & & \\
$1.00000 \mathrm{E}-01$ & $1.954 \mathrm{E}+00$ & $1.502 \mathrm{E}+00$ \\
$1.15606 \mathrm{E}-01$ & $1.378 \mathrm{E}+00$ & $1.027 \mathrm{E}+00$ \\
$1.15606 \mathrm{E}-01$ & $4.893 \mathrm{E}+00$ & $1.382 \mathrm{E}+00$ \\
$1.50000 \mathrm{E}-01$ & $2.591 \mathrm{E}+00$ & $1.083 \mathrm{E}+00$ \\
$2.00000 \mathrm{E}-01$ & $1.298 \mathrm{E}+00$ & $6.746 \mathrm{E}-01$ \\
$3.00000 \mathrm{E}-01$ & $5.192 \mathrm{E}-01$ & $3.050 \mathrm{E}-01$ \\
$4.00000 \mathrm{E}-01$ & $2.922 \mathrm{E}-01$ & $1.732 \mathrm{E}-01$ \\
$5.00000 \mathrm{E}-01$ & $1.976 \mathrm{E}-01$ & $1.152 \mathrm{E}-01$ \\
$6.00000 \mathrm{E}-01$ & $1.490 \mathrm{E}-01$ & $8.494 \mathrm{E}-02$ \\
$8.00000 \mathrm{E}-01$ & $1.016 \mathrm{E}-01$ & $5.574 \mathrm{E}-02$ \\
$1.00000 \mathrm{E}+00$ & $7.896 \mathrm{E}-02$ & $4.241 \mathrm{E}-02$ \\
$1.25000 \mathrm{E}+00$ & $6.370 \mathrm{E}-02$ & $3.351 \mathrm{E}-02$ \\
$1.50000 \mathrm{E}+00$ & $5.587 \mathrm{E}-02$ & $2.891 \mathrm{E}-02$ \\
$2.00000 \mathrm{E}+00$ & $4.878 \mathrm{E}-02$ & $2.523 \mathrm{E}-02$ \\
$3.00000 \mathrm{E}+00$ & $4.447 \mathrm{E}-02$ & $2.434 \mathrm{E}-02$ \\
$4.00000 \mathrm{E}+00$ & $4.392 \mathrm{E}-02$ & $2.546 \mathrm{E}-02$ \\
$5.00000 \mathrm{E}+00$ & $4.463 \mathrm{E}-02$ & $2.689 \mathrm{E}-02$ \\
$6.00000 \mathrm{E}+00$ & $4.583 \mathrm{E}-02$ & $2.829 \mathrm{E}-02$ \\
$8.00000 \mathrm{E}+00$ & $4.879 \mathrm{E}-02$ & $3.068 \mathrm{E}-02$ \\
$1.00000 \mathrm{E}+01$ & $5.195 \mathrm{E}-02$ & $3.259 \mathrm{E}-02$ \\
$1.50000 \mathrm{E}+01$ & $5.927 \mathrm{E}-02$ & $3.552 \mathrm{E}-02$ \\
$2.00000 \mathrm{E}+01$ & $6.512 \mathrm{E}-02$ & $3.662 \mathrm{E}-02$
\end{tabular}

Table 1. The mass-density normalized gamma-ray exponential attenuation coefficient in uranium as a function of gamma-ray energy (data of Figure 1 ). $\mu_{\text {en }}$ is the attenuation due to the atomic electron interactions only, $\mu$ is the total attenuation. 


\begin{tabular}{|c|c|c|c|}
\hline \multirow{2}{*}{$\begin{array}{c}\text { Momentum } \\
\text { interval } \\
\mathrm{GeV} / \mathrm{c}\end{array}$} & \multirow{2}{*}{$\begin{array}{c}\text { Mean } \\
\text { momentum } \\
\mathrm{GeV} / \mathrm{c}\end{array}$} & \multicolumn{2}{|c|}{$\begin{array}{l}\text { CALRICE94 } \\
\left(\mathrm{GeV} / \mathrm{cm}^{2} \mathrm{~s} s\right)^{-1}\end{array}$} \\
\hline & & $\mu^{+}$ & $\mu^{-}$ \\
\hline $02-0.3$ & 0.25 & $(1.4 \pm 0.2) \times 10^{1}$ & $(1.1 \pm 0.1) \times 10^{1}$ \\
\hline $0.3-0.4$ & 0.35 & $(1.68 \pm 0.08) \times 10^{1}$ & $(136 \pm 0.07) \times 10^{1}$ \\
\hline $0.40-0.55$ & 0.47 & $(1.72 \pm 0.05) \times 10^{1}$ & $(1.44 \pm 0.04) \times 10^{1}$ \\
\hline $0.55-0.70$ & 0.62 & $(1.66 \pm 0.04) \times 10^{1}$ & $(135 \pm 0.03) \times 10^{1}$ \\
\hline $0.70-0.85$ & 0.78 & $(1.56 \pm 004) \times 10^{1}$ & $(133 \pm 0.03) \times 10^{1}$ \\
\hline $0.85-1.0$ & 0.92 & $(1.48 \pm 0.03) \times 10^{1}$ & $(121 \pm 0.03) \times 10^{1}$ \\
\hline $10-1.2$ & 1.1 & $(1.30 \pm 0.03) \times 10^{1}$ & $(1.10 \pm 0.03) \times 10^{1}$ \\
\hline $12-1.4$ & 1.3 & $(1.20 \pm 0.03) \times 10^{1}$ & $(101 \pm 0.02) \times 10^{1}$ \\
\hline $1.4-1.6$ & 1.5 & $102 \pm 02$ & $8.7 \pm 0.2$ \\
\hline $1.6-2.1$ & 1.84 & $9.1 \pm 02$ & $7.3 \pm 0.1$ \\
\hline $21-2.94$ & 2.49 & $6.6 \pm 0.1$ & $5.20 \pm 0.09$ \\
\hline $2.94-4.12$ & 3.49 & $4.12 \pm 007$ & $3.38 \pm 0.06$ \\
\hline $4.12-5.5$ & 4.78 & $2.53 \pm 005$ & $1.98 \pm 0.04$ \\
\hline $55-70$ & 6.21 & $1.61 \pm 0.03$ & $1.25 \pm 0.03$ \\
\hline $70-100$ & 8.37 & $(9.0 \pm 0.2) \times 10^{-1}$ & $(69 \pm 01) \times 10^{-1}$ \\
\hline $100-1.5 .5$ & 12.42 & $(3.89 \pm 0.08) \times 10^{-1}$ & $1309 \pm 0.07) \times 10^{-1}$ \\
\hline $15 s-230$ & 18.85 & $(1.38 \pm 0.04) \times 10^{-1}$ & $(1.08 \pm 0.03) \times 10^{-1}$ \\
\hline $230-31.1$ & 26.68 & $(6.3 \pm 0.3) \times 10^{-2}$ & $(4.6 \pm 0.2) \times 10^{-2}$ \\
\hline $31-1-43.6$ & 36.69 & $(2.8 \pm 0.1) \times 10^{-1}$ & $(1.9 \pm 01) \times 10^{-2}$ \\
\hline $43.6-61.1$ & 51.47 & $(9.9 \pm 0.7) \times 10^{-3}$ & $(7.1 \pm 0.6) \times 10^{-1}$ \\
\hline $61.1-85.6$ & 72.08 & $(3.6 \pm 0.3) \times 10^{-3}$ & $(30 \pm 0.3) \times 10^{-3}$ \\
\hline $85.6-1200$ & 100.96 & $(1.4 \pm 0.2) \times 10^{-3}$ & $(12 \pm 02) \times 10^{-3}$ \\
\hline
\end{tabular}

Table 2. The sea-level muon flux,
separated into charge components.

${ }^{8}$ From J. Kremer et al., Phys. Rev. Lett. 83 (1999) 4241. 


\begin{tabular}{|c|c|c|c|c|c|c|}
\hline \multirow[b]{2}{*}{ Transition } & \multicolumn{2}{|c|}{ This experiment ${ }^{\mathrm{a}}$} & \multicolumn{2}{|c|}{ This calculation } & \multicolumn{2}{|c|}{ CERN calculation $^{b}$} \\
\hline & Energy (ke V) & Rel. int. & Energy (kev) & Rel. int. & Energy (ke V) & Rel, int. \\
\hline \multirow[t]{25}{*}{$2 p-1 s$} & $6112.45 \pm 2.45$ & 0.009 & & & & \\
\hline & & & 6117.76 & 0.010 & 6118.7 & 0.018 \\
\hline & $* 6119.86 \pm 1.40$ & 0.020 & 6121.71 & 0.018 & 6121.1 & 0.017 \\
\hline & $* 6150.11 \pm 0,87$ & 0.035 & 6150.63 & 0.025 & & \\
\hline & ${ }^{*} 6158.09+1.26$ & 0.078 & 6157.57 & 0.054 & 6156.9 & 0.057 \\
\hline & $* 6165.23 \pm 1,22$ & 0.211 & 6163.96 & 0.131 & 6163.2 & 0.136 \\
\hline & & & 6167.91 & 0.139 & 6167.1 & 0.142 \\
\hline & $* 6169.77 \pm 1.15$ & 0.118 & 6170.83 & 0.049 & & \\
\hline & & & & & 6175.7 & 0.054 \\
\hline & & & & & 6181.8 & 0.020 \\
\hline & ${ }^{*} 6205.82+0.86$ & 0.029 & 6203.77 & 0.016 & & \\
\hline & & & 6207.43 & 0.011 & & \\
\hline & $* 6397.96 \pm 1.18$ & 0.023 & 6397.77 & 0.020 & 6395.8 & 0.020 \\
\hline & & & 6414,37 & 0.015 & 6413.3 & 0.026 \\
\hline & $* 6414,65 \pm 1,17$ & 0.048 & 6415.15 & 0.028 & & \\
\hline & $-6453.06 \pm 1.31$ & 0.042 & 6454.57 & 0.023 & 6452.8 & 0.022 \\
\hline & & & 6460.57 & 0.090 & 6458.6 & 0.087 \\
\hline & ${ }^{*} 6460.36 \pm 1.24$ & 0.215 & 6461,34 & 0.015 & & \\
\hline & & & 6461.35 & 0.104 & 6459.3 & 0.098 \\
\hline & & & 6484.63 & 0.013 & & \\
\hline & ${ }^{*} 6501.63 \pm 1.36$ & 0.033 & 6500.77 & 0.032 & 6498.8 & 0.030 \\
\hline & & & & & 6523.1 & 0.019 \\
\hline & ${ }^{*} 6544.28 \pm 1,39$ & 0.024 & 6546.23 & 0.014 & & \\
\hline & $+6557,36=1.45$ & 0.098 & 6558,58 & 0.085 & 6556.4 & 0.082 \\
\hline & ${ }^{*} 6587.44 \pm 2.82$ & 0.018 & 6587.63 & 0.013 & & \\
\hline \multirow[t]{26}{*}{$3 d-2 p$} & $+2910.80 \neq 0.42$ & 0.022 & 2910.96 & 0.016 & & \\
\hline & $* 2946.48 \pm 0.40$ & 0.036 & 2945.69 & 0.022 & 2946.7 & 0.025 \\
\hline & $* 2959.47 \pm 0.38$ & 0.044 & 2960.28 & 0.030 & 2961.4 & 0.037 \\
\hline & $* 2984.86 \pm 0.46$ & 0.028 & 2984.28 & 0.015 & & \\
\hline & $* 2997.44 \pm 0.87$ & 0.062 & 2997,81 & 0.053 & 2998.7 & 0.055 \\
\hline & $3016.54 \pm 0.80$ & 0.022 & & & & \\
\hline & & & 3021.24 & 0.036 & 3022.0 & 0.037 \\
\hline & $* 3023.52 \div 0.79$ & 0.171 & 3022.03 & 0.024 & 3022.7 & 0.025 \\
\hline & & & 3025.13 & 0.067 & 3026.1 & 0.069 \\
\hline & & & 3031.86 & 0.010 & & \\
\hline & $+3032,21 \pm 0.46$ & 0.051 & 3032.65 & 0.021 & 3033.5 & 0.022 \\
\hline & $* 3043.73 \div 0.44$ & 0.041 & 3043.71 & 0.027 & 3044.5 & 0.030 \\
\hline & $+3211.42 \pm 0.52$ & 0.018 & 3212.03 & 0.015 & 3212.7 & 0.026 \\
\hline & $* 3224.93+0.51$ & 0.022 & 3225.29 & 0.026 & 3225.2 & 0.024 \\
\hline & $* 3242,06 \pm 0.52$ & 0.096 & 3241.96 & 0.063 & 3241.7 & 0.056 \\
\hline & & & 3244.96 & 0.033 & & \\
\hline & $\star 3246.67=0.58$ & 0.124 & 3245.91 & 0.052 & 3245.6 & 0.046 \\
\hline & & & 3247.63 & 0.028 & 3247,4 & 0.025 \\
\hline & $* 3252.64=0.53$ & 0.070 & 3251.58 & 0.050 & 3251.3 & 0.045 \\
\hline & & & 3259.18 & 0.014 & & \\
\hline & $* 3260.47 \pm 0.45$ & 0.090 & 3261.15 & 0.066 & 3261.0 & 0.063 \\
\hline & & & 3265,03 & 0.014 & 3263.8 & 0.023 \\
\hline & $3267,56=0.50$ & 0.035 & 3269.45 & 0.021 & & \\
\hline & & & 3275.44 & 0.016 & & \\
\hline & $* 3281.21=0.45$ & 0.050 & 3280.89 & 0.036 & 3280.7 & 0.037 \\
\hline & $3317.87 \pm 0.55$ & 0.015 & & & & \\
\hline
\end{tabular}

Table 3. The measured muonic X-ray energies and relative intensities ${ }^{9}$ from $\mathrm{HEU}$ for the lowest two sets of transitions. Also shown are two theoretical calculations.

${ }^{9}$ Adapted from D. A. Close, J. J. Malanify, and J. P. Davidson, Phys. Rev. C17 (1978) 1433. 


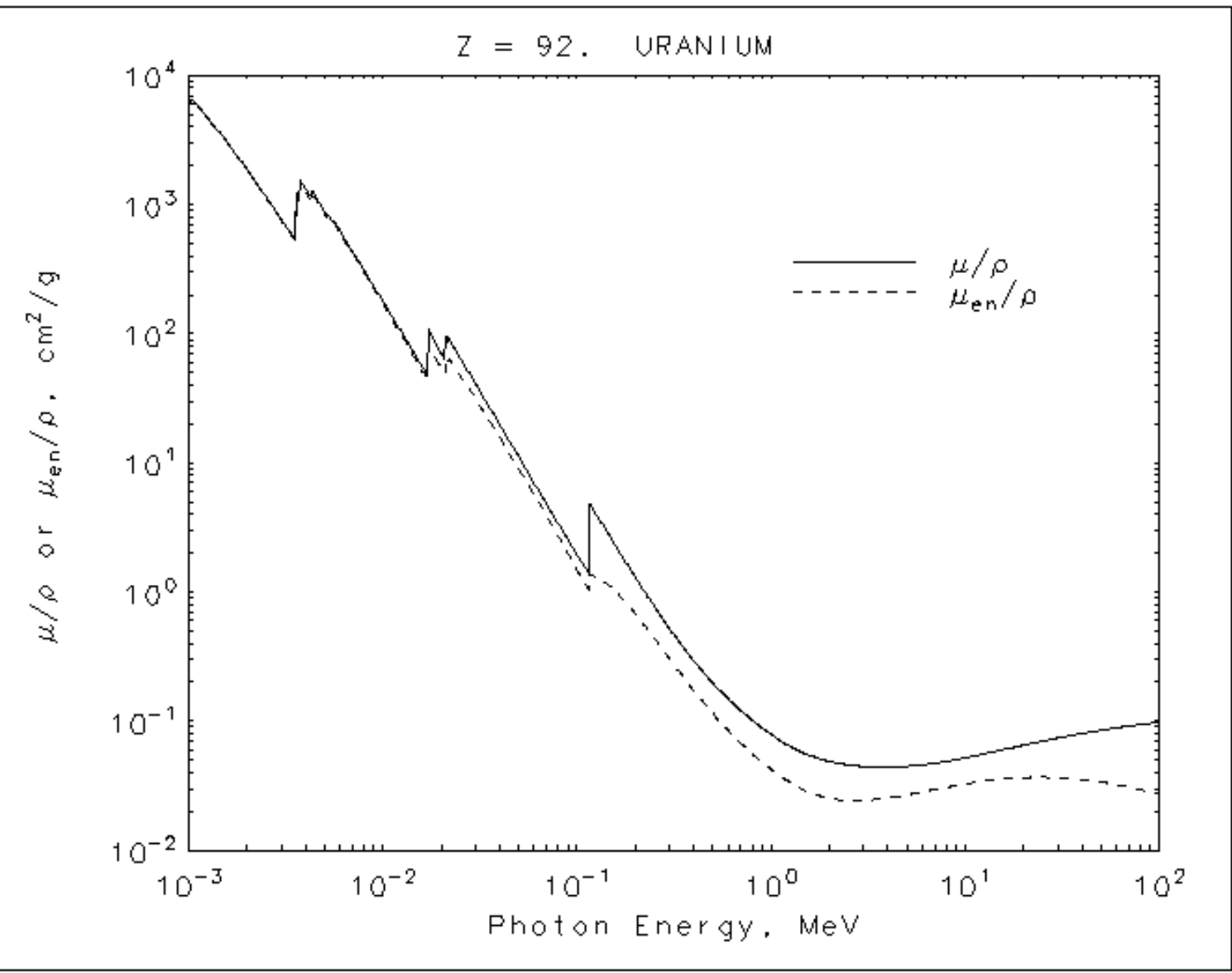

Figure 1. The mass-density normalized gamma-ray exponential attenuation coefficient in uranium as a function of gamma-ray energy. ${ }^{10} \mu_{\mathrm{en}}$ is the attenuation due to the atomic electron interactions only, $\mu$ is the total attenuation.

${ }^{10}$ From NIST, Physical Reference Data, X-ray mass attenuation coefficients. 


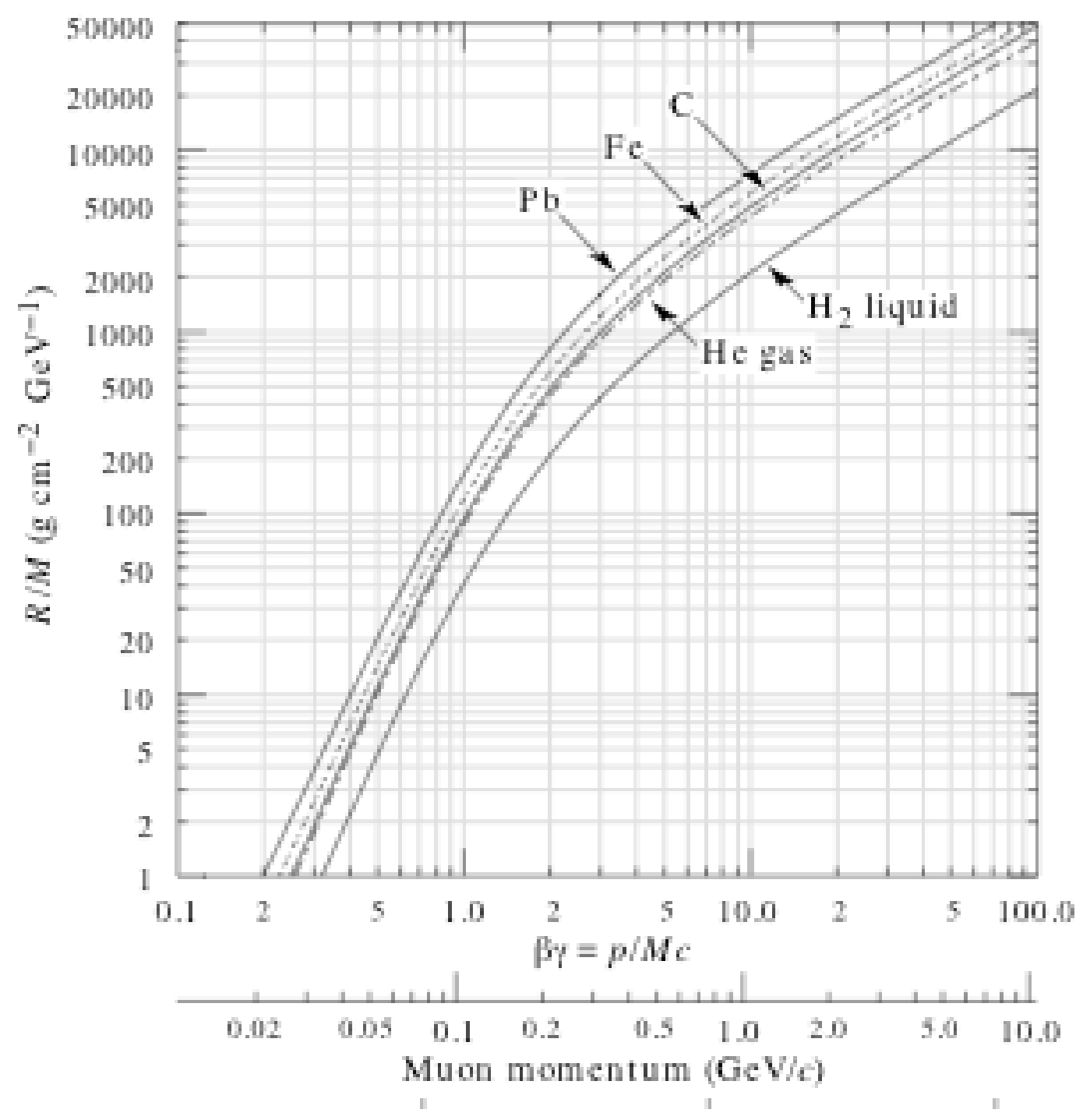

Figure 2. The range of muons in units of target mass density times projectile mass as a function of muon momentum. ${ }^{11}$

${ }^{11}$ From Particle Data Group (LBL) compilation, 2003. 


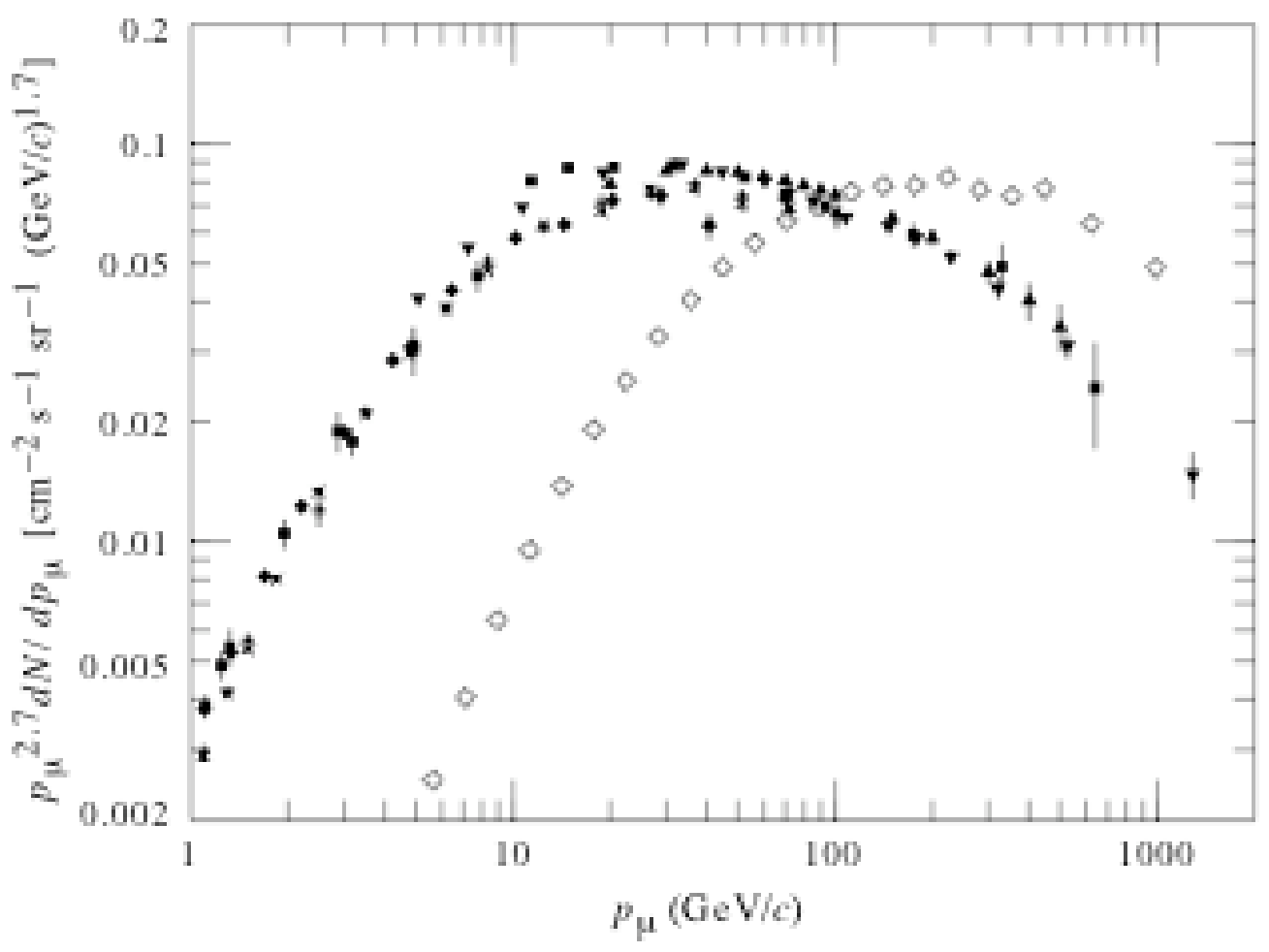

Figure 3. The sea-level muon energy, scaled by a power of the muon momentum to flatten the curve. ${ }^{12}$ The solid points are for vertical muons, the hollow points are for steeply-arriving muons.

\footnotetext{
${ }^{12}$ Particle Data Group (LBL) compilation, 2003.
} 

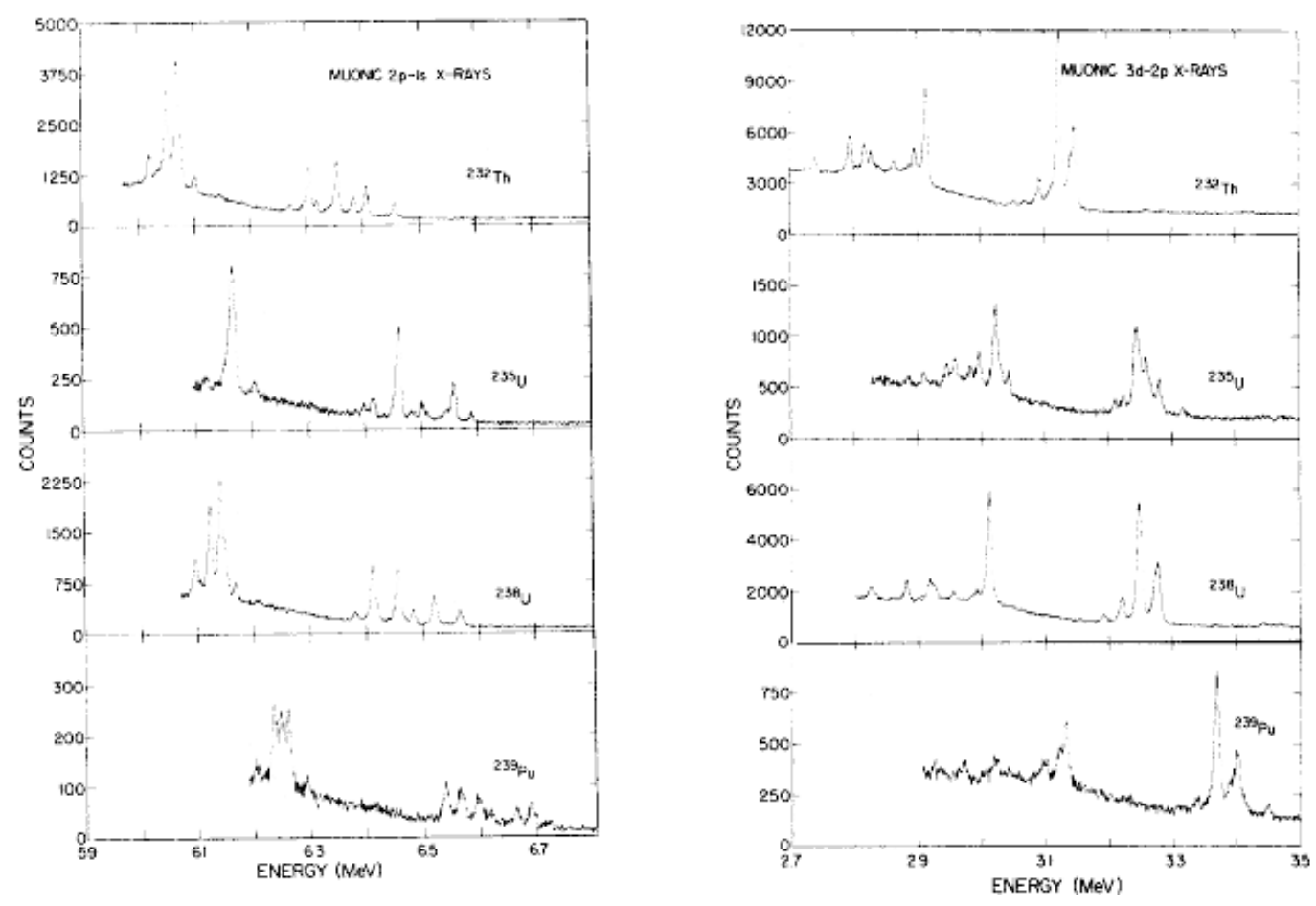

Figure 4. The measured $2 \mathrm{p}-1 \mathrm{~s}$ and $3 \mathrm{~d}-2 \mathrm{p}$ muonic X-ray spectra (from Table 3 ) for a range of actinides, including $\mathrm{HEU}$ and $\mathrm{Pu}$. 


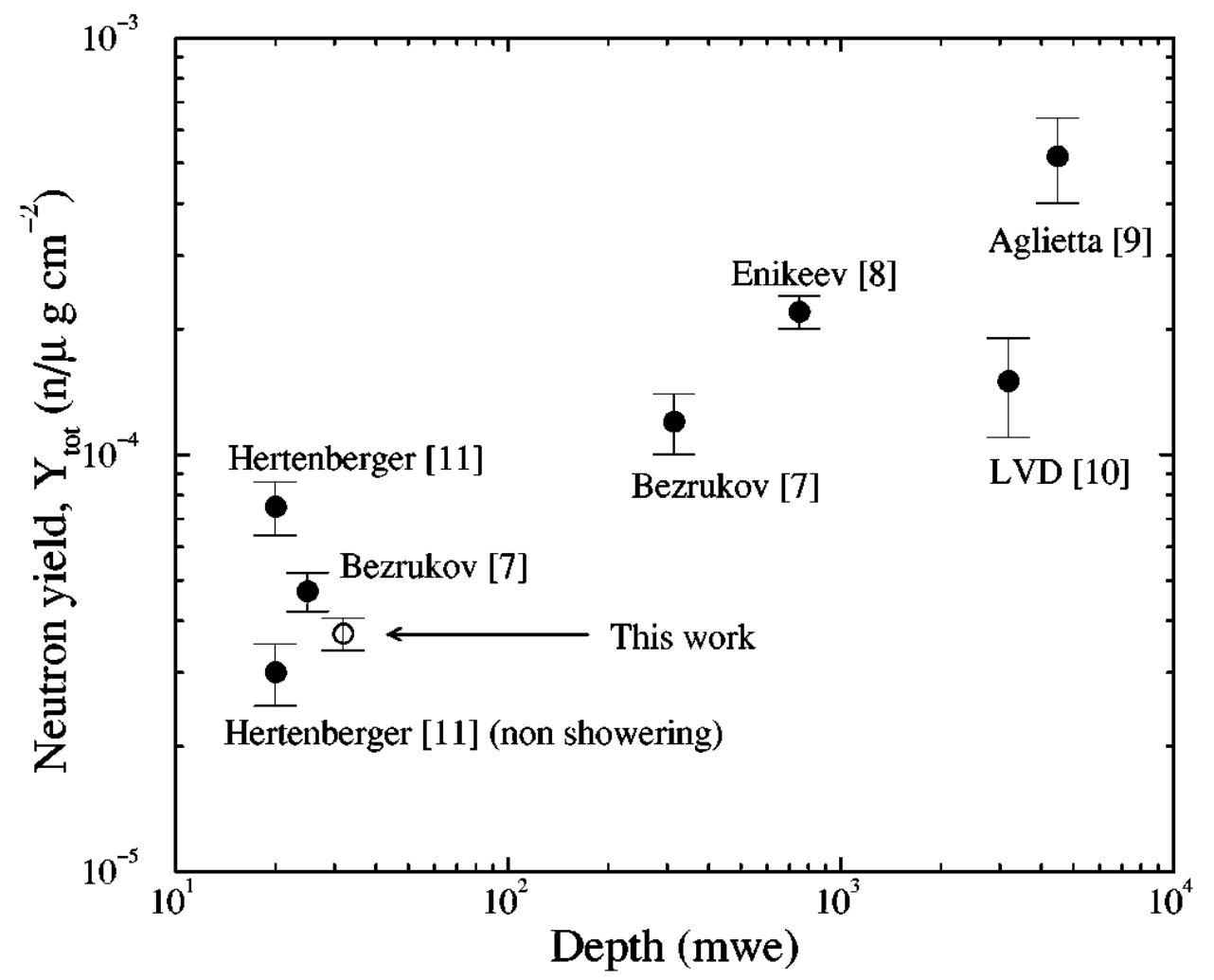

Figure 5. The total neutron yield at various depths (neutrons per cosmic ray per target mass) from Boehm et al. The target in their work is liquid scintillator. 


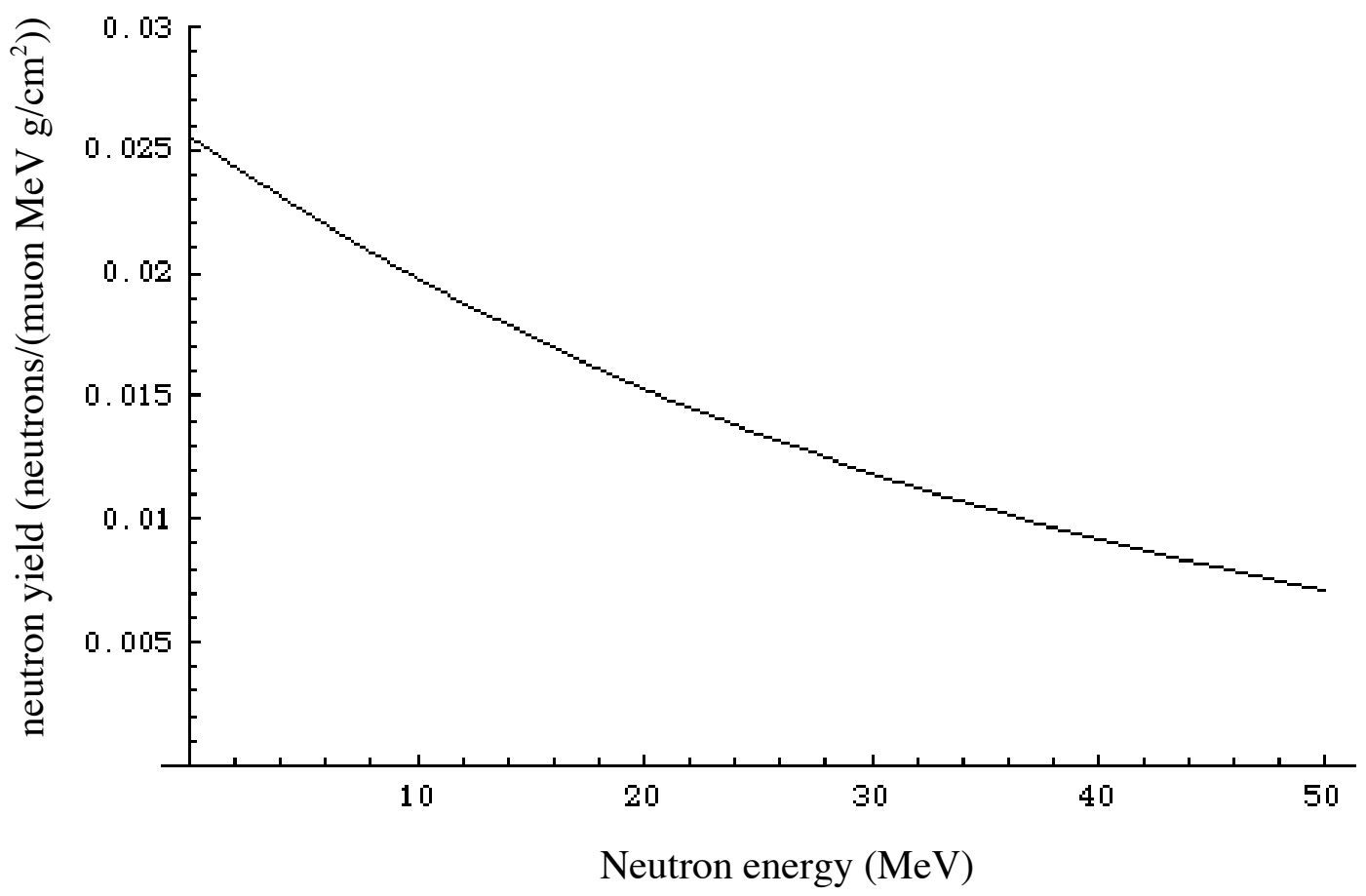

Figure 6. The Karmen neutron energy distribution normalized to the extrapolated sea-level Boehm et al. neutron yield. 


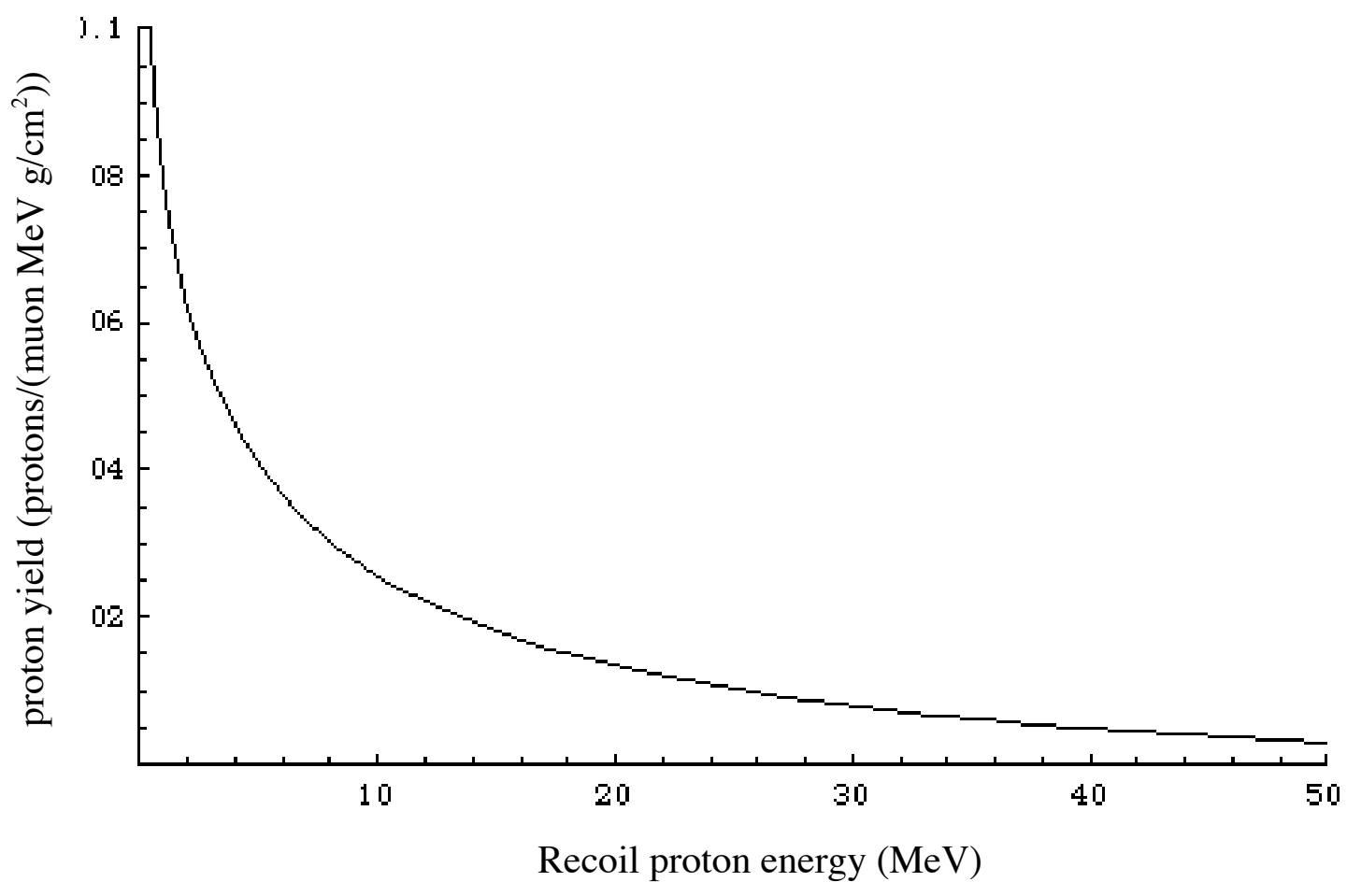

Figure 7. The proton recoil yield from the neutrons associated with cosmic-ray muons. 